\title{
Corela
}

Cognition, représentation, langage

HS-8 | 2010

L'interpellation

\section{L'interpellation en français : un système comme les autres?}

Reyes Leon Miranda

\section{(2) OpenEdition}

Journals

Édition électronique

URL : http://journals.openedition.org/corela/1824

DOI : $10.4000 /$ corela. 1824

ISSN : 1638-573X

Éditeur

Cercle linguistique du Centre et de l'Ouest - CerLICO

Référence électronique

Reyes Leon Miranda, "L'interpellation en français : un système comme les autres? », Corela [En ligne], HS-8 | 2010, mis en ligne le 24 novembre 2010, consulté le 30 avril 2019. URL : http:// journals.openedition.org/corela/1824; DOI : 10.4000/corela.1824

Ce document a été généré automatiquement le 30 avril 2019.

\section{(c) (i) (2)(2)}

Corela - cognition, représentation, langage est mis à disposition selon les termes de la licence Creative Commons Attribution - Pas d'Utilisation Commerciale - Partage dans les Mêmes Conditions 4.0 International. 


\title{
L'interpellation en français : un système comme les autres?
}

\author{
Reyes Leon Miranda
}

\section{Terminologie}

1 En ce qui concerne mon étude sur les formes d'adresse et d'interpellation, je retiendrai les deux expressions suivantes : forme nominale d'adresse et forme mimo-gestuelle d'adresse.

2 J'emprunte à C. Kerbrat-Orecchioni (2010, à paraître) le terme forme nominale d'adresse (dorénavant FNA) qu'elle et son équipe de recherche $^{1}$ ont choisi pour référer aux syntagmes nominaux (noms, pronoms, adjectifs nominalisés, et leurs combinaisons) qui posent «explicitement, face à l'énonceur [...] celui que ce dernier a choisi comme destinataire » (Serbat, 1987) et qui remplissent les fonctions discursives que A. M. Bañon (1993) attribue au vocatif et C. Détrie (2006) à l'apostrophe. Sur mes corpus, je n'étudierai que les noms ou adjectifs nominalisés car « ces différentes catégories [...] sont en principe chargées de valeurs sémantiques plus précises que ces indications de distance et hiérarchie que comportent seulement, et éventuellement, les pronoms d'adresse » (Kerbrat-Orecchioni, 1992, p. 47).

3 Partant de cette définition, sont exclus de mon concept de FNAles interjectionsen tant que noms prédicatifs exclamatifs dont Wagner et Pinchon (1991, p. 82) nous fournissent les exemples suivants : «Silence! Attention!».

4 Je retiendrai également l'expression forme mimo-gestuelle d'adresse (FMGA par la suite) -qui reflète une indiscutable cohérence par rapport à celle de forme nominale d'adressepour renvoyer aux signes non verbaux et non vocaux, c'est-à-dire aux gestes et mimiques, qui posent «explicitement, face à l'énonceur [...] celui que ce dernier a choisi comme destinataire » (Serbat, 1987) et qui remplissent les fonctions discursives que A. M. Bañon (1993) attribue au vocatif et C. Détrie (2006) à l'apostrophe. D'ailleurs, cette dernière prend déjà en compte la dynamique corporelle de changement positionnel et d'orientation vers l'apostrophé contenue sémantiquement dans le mot apostrophe: 
5 Le mot, dans sa morphologie inscrit une problématique déjà corporelle : apo- construit la valeur $d$ "éloignement, et -strophe, celle de tour, soit un tour qui détourne du développement principal dans le but d'interpeller autrui [...], l'apostrophe s'accompagnant d'une orientation corporelle vers celui qu'on interpelle, soit une figure d'interpellation, liée au face à face (2006, p. 16).

En ce qui concerne ma notion de $F M G A$, je l'inscrirai dans cette dimension corporelle du langage qui fournit des renseignements qui sont absents de la chaîne parlée ou vocalisée.

En résumé, j'emploierai le terme FNA pour tout appel à l'interlocuteur émis à l'aide du langage verbal, et notamment des syntagmes nominaux, et le terme FMGA saura être son homologue dans le langage non verbal et non vocal.

\section{Une crise d'appellatifs}

8 Catherine Kerbrat-Orecchioni lançait déjà en 1992 l'idée que le français subissait une crise d'appellatifs. Pour arriver à cette conclusion, elle avait parcouru les études que d'autres auteurs avaient réalisées auparavant sur les systèmes d'adresse de nombreuses langues. La compréhension et la comparaison du fonctionnement des différents systèmes appellatifs lui permettent de remarquer qu'il y a des langues

où il est «quasiment impossible » de dire quoi que ce soit sans spécifier du même coup le type de relation que l'on entend entretenir avec son partenaire d'interaction: dans bien des sociétés, le système linguistique est ainsi fait que sa mise en discours entraîne automatiquement l'indexation mutuelle des interlocuteurs. (ibid., p. 34)

9 En revanche, l'indexation mutuelle des interlocuteurs présuppose l'accessibilité et le partage immédiats et réciproques des interlocuteurs à certaines informations d'ordre personnel, relationnel et interrelationnel telles que :

«leur sexe, leur âge, leur statut social», leur «degré de connaissance, le type de lien » qui les unit, «l'appartenance éventuelle à un même groupe, [...] le type de contrat qui les lie durant l'échange communicatif, [...] le nombre de participants et le caractère plus ou moins formel de la situation d'interaction ». (ibid., p. 36)

10 À ce sujet Kerbrat-Orecchioni explique que, si tous ces paramètres jouent un rôle dans toutes sortes d'interactions verbales de n'importe quelle société, «ce qui change d'une société à l'autre, c'est leur importance relative, leur hiérarchie, et les phénomènes de neutralisation qui en découlent » (ibid., p. 36).

En espagnol, par exemple, dans certains emplois des FNA le paramètre du sexe de l'interlocuteur est privilégié au détriment de paramètres tels que l'âge ou le statut social couramment neutralisés: niña, niño, chica, chico (fille, garçon $\rightarrow$ féminin vs masculin) peuvent se voir appliqués pour appeler des femmes ou des hommes à l'âge adulte. Dans ce cas, ces FNA véhiculent parallèlement quelques traits apparentés au référent primaire (ici, l'enfant) qui éveillent chez le destinataire une représentation précise d'un comportement, d'un statut, d'une idée argumentative, etc. appartenant à l'imaginaire collectif (R. León Miranda, 2011, en préparation). Souvent, suite à la désémantisation du mot, même le paramètre du sexe est neutralisé et l'on peut s'adresser à une femme en lui disant : hombre (homme) (ibid.).

12 Pour revenir au cas du français, Kerbrat-Orecchioni considère toutes les prémisses exposées ci-dessus et l'absence de FNA dans les corpus oraux de conversations naturelles dont elle avait fait l'étude, pour finalement introduire la notion «d'appellatif zéro » 
qu'elle applique au système français. À l'époque, elle avait défini l'appellatif zéro comme étant «le choix préférentiel en cas d'ambiguïté relationnelle» (1992, p. 55). Conséquemment, l'absence d'interpellation nominale en français pourrait se justifier, selon Kerbrat-Orecchioni, par la tentative de contourner une situation socialement embarrassante provoquée notamment par:

le manque "des informations nécessaires au choix de la forme appropriée » pour interpeller l'écouteur, ou bien, par le défaut «d'une forme d'adresse [...] véritablement appropriée à la circonstance » (ibid., p. 54).

13 Ainsi, la mise en pratique de l'appellatif zéro en tant qu'attitude linguistique constituerait le facteur déclencheur de la crise d'appellatifs en français :

face à l'aporie, et à l'impossibilité de trouver une solution élégante, le locuteur adopte une prudente stratégie d'évitement; en omettant l'appellatif il échappe au risque d'impair, et s'épargne un choix qui peut être délicat. (ibid.)

14 Cette attitude linguistique dévoilerait une problématique à caractère sociolinguistique si c'est l'individu-locuteur qui hésite à employer un terme ou un autre par manque d'informations sur son destinataire. D'un autre côté, la crise d'appellatifs pourrait répondre également à une question d'ordre lexicologique si c'est justement le manque de terme adéquat ce qui coince le locuteur à émettre son appel à l'aide des FNA. D'ailleurs, cette crise pourrait résulter tout simplement de la combinaison des facteurs sociolinguistique et lexicologique exposés ci-dessus.

15 À cet égard, R. ENAJAS (2004) explique comment le facteur culturel conditionne les centres d'intérêt d'une communauté linguistique et la création conséquente du répertoire lexical nécessaire, système d'adresse inclus, pour y référer ${ }^{2}$.

16 C'est bien connu que la disponibilité lexicale d'une communauté linguistique dépend, entre autres facteurs, de ses centres d'intérêt social, politique, économique, personnel, etc. On entend assez souvent que les hanoo des Philippines ont 92 noms pour désigner le riz, ou que les Esquimaux en ont autant pour les différents types de neige. Si l'on transpose cela au plan des systèmes d'adresse, on pourra observer comment les relations interpersonnelles donnent lieu, selon le degré d'intimité, à de nouvelles formes d'appeler nos proches basées sur le prénom ou inspirées par un autre type d'association.

17 Par rapport au répertoire lexical du système d'adresse français Kerbrat-Orecchioni précise que « en français comme en anglais, le système des marqueurs relationnels est donc assurément, par comparaison, très indigent, mais il n'est pas pour autant inexistant ». (1992, p. 34)

De mon point de vue et d'après les arguments que je fournis par la suite, l'usage de l'appellatif zéro se développerait aussi à partir de l'association établie instinctivement entre les FNA et la fonction conative.

Locutrice espagnole de langue maternelle espagnole, une fois en France et mise en situation d'immersion linguistique, j'ai constaté l'indigence lexicale du système d'adresse français. Dans mes débuts en français oral et interactionnel, $\mathrm{j}$ 'essayais sans succès de transposer en français le système d'adresse espagnol: je me devais d'inclure mes interlocuteurs dans la conversation et de les appeler constamment pour accomplir, entre autres, les fonctions discursives phatique (vérification d'ouverture et du maintien du canal communicatif) et argumentative. Alors, je me suis résolue à utiliser les noms de mes interlocuteurs (noms de famille, prénoms, surnoms, diminutifs) pour baliser avec eux mes interventions. Mais ce comportement verbal encombrait mes énonciations et contrariait 
(linguistiquement parlant) mes interlocuteurs qui, eux, ne s'attendaient pas, par habitude et par usage, à être interpellés à tout bout de champ. Cette contrariété linguistique de mes écouteurs était légitimée parce que mes interpellations déclenchaient en eux des attentes d'une suite différente du discours plutôt orientée vers la sollicitation, la demande, la prière, etc. Ce phénomène d'attente instinctive d'une suite discursive déterminée est expliqué par D. Seleskovitch et L. Lederer (2001, p. 276) à partir des techniques appliquées en interprétation simultanée et il correspond à la façon dont on appréhende le sens du discours par anticipation d'unités de sens.

Les unités de sens sont le produit d'une synthèse de quelques mots qui se trouvent dans la mémoire immédiate et des expériences ou des souvenirs cognitifs préexistants qu'ils éveillent. [...] Il convient, lorsqu'on étudie les anticipations, d'établir une nette distinction entre celles du sens et celles des mots de la langue. (ibid.)

21 La prévision de mots est un phénomène connu en traduction et en interprétation sous le nom de collocation et consiste à appréhender «le contenu sémantique de mots non encore prononcés mais annoncés par d'autres mots que l'on a l'habitude de lire ou d'entendre ensemble » (ibid.). Pour ce qui est de l'anticipation du sens, en revanche, «ce n'est plus alors l'habitude d'associer des mots qui permet d'anticiper, mais l'habitude d'associer des connaissances" (ibid.). En somme, l'anticipation de sens serait l'équivalent de la collocation au niveau du discours. Or, je suis persuadée que c'est justement ce processus d'association de connaissances qui décontenançait mes interlocuteurs français lorsque je les interpellais sans cesse.

En effet, en français oral l'occurrence des FNA annonce notamment deux opérations pragmatiques précises :

- premièrement, l'utilisation des FNA est liée aux salutations du début ou de la fin d'une interaction verbale. Cette idée est corroborée par l'analyse de Dimachki \& Hmed (2001). Les moments d'apparition privilégiés des termes d'adresse dans le corpus français sont ceux des salutations d'ouverture et de clôture. Dans ces interactions, l'accompagnement des salutations par un terme d'adresse est d'ailleurs quasi-rituel.

- deuxièmement, en français oral, l'appel à l'allocutaire construit par les FNA est couramment associé à une prière, à une sollicitation ou à un ordre. D. Lagorgette (citée par C. Détrie, 2006, p.118) ne manque pas de souligner que les FNA provoquent chez les adressés l'attente d'une suite discursive chargée de force illocutoire.

Les T.A. [termes d'adresse] sont très fortement représentés dans les actes illocutoires : demande, ordre, mais aussi excuses, et généralement toute forme de négociation explicite. [...] on peut dire de manière plus générale que là où l'on trouve des termes d'adresse, on doit s'attendre à découvrir sous peu une situation FTA.

En français, la présence des FNA dans le discours oral surtout de type familier (en dehors des protocoles figés par les cadres institutionnels) serait tout de suite associée par anticipation de sens à des actes d'ouverture ou de clôture de la communication, de politesse (remerciements, excuses) et de sollicitation (prières, ordres, questions).

En milieu d'interaction la possibilité concernant les salutations reste exclue ; et mis à part les remerciements qui constituent une réponse à une manifestation de la part de l'autre, le reste du temps (la plupart du temps) on appelle quand nous avons besoin de quelque chose. De toute évidence, lorsque l'on émet un appel, on attend toujours une réponse. Un appel n'est jamais lancé gratuitement: sa réalisation cherche à atteindre un but, un signal, une réaction de la part de l'autre. Du moment où l'on appelle un interlocuteur, on 
le «force", pour ainsi dire, à réagir. On sollicite de lui qu'il nous regarde, qu'il nous écoute, qu'il nous réponde, qu'il nous accorde le tour de parole, qu'il effectue une démarche quelconque, etc.

En outre, l'appel suppose également une «menace pour la face » (E. Goffman, 1974) de son émetteur. Comme on l'a précédemment évoqué, il faut avoir besoin d'atteindre quelque chose pour appeler et l'émetteur de l'appel en est conscient. Il espère en quelque sorte; il dévoile une faiblesse face à son interlocuteur, une dépendance. Ce sentiment le rend donc vulnérable et il doit alors faire un choix :

- soit il lance l'appel mais il n'utilise aucune FNA (appellatif zéro) pour donner la fausse impression que son énoncé, bien qu'adressé à son destinataire, n'a pas une visée exclusivement conative. Ici, l'absence de FNA pourrait s'interpréter comme la volonté d'adoucir la force illocutoire de l'énoncé. Certes si un énoncé à visée conative se voit doublé par l'effet d'une FNA associée instinctivement elle aussi à la fonction conative, cela risque d'être pris pour une vraie «menace » au lieu d'un simple appel.

- soit il lance l'appel et il ose utiliser la FNA en avouant explicitement être à la quête d'une réponse.

Dans les deux cas, on verra comment l'appel (verbal ou mimo-gestuel) se réalise.

En somme, pour expliquer la crise de FNA en français oral familier, trois facteurs ont été retenus, à savoir le manque d'informations sur l'interlocuteur, le manque de terme approprié, et l'association des FNA à la fonction conative.

Étant donné que nous ne disposons pas de corpus oraux plus anciens pour mener à bien une étude diachronique, nous ignorons si cette crise de FNA est la conséquence directe d'un de ces facteurs, de la synergie des trois ou peut-être d'aucun d'entre eux. Il se peut aussi que le style communicatif $d u$ français oral familier ait évolué vers un style communicatif moins interjectif (L. Pop, 2006)ou, tout au contraire, qu'il ait toujours été ainsi.

\section{Un style communicatif moins interjectif ?}

Dans une étude comparative sur les styles communicatifs français et roumain, L. Pop (ibid. )montre que le français est une langue moins encline à l'utilisation des interjectifs que le roumain. Pour les mêmes fonctions discursives assurées en français par les « marqueurs de structuration de conversation " (ibid., p. 25) dont notamment eh bien, le roumain met en jeu des interjections appellatives privilégiant «un mode de communication dirigé vers l'autre » (ibid., p. 34). Parmi les «marqueurs d'attention » (ibid., p. 26) repérés dans les corpus roumains comme «synonymes discursifs ou équivalents fonctionnels » (ibid., p. 26) de eh bien, L. Pop distingue les "phatiques » ou «marqueurs d'appel, à l'aide desquels le locuteur attire l'attention de son interlocuteur sur des informations importantes » (ibid., p. 26). Entre autres, elle signale des «substituts du pronom tu [...], des appellatifs nominaux dont certains se sont désémantisés [...] et des formes combinées (pronoms+substantifs) [...] ou syntagmes nominaux" (ibid., p. 26) investis d'une fonction vocative dominante. L. Pop montre également comment les expressions injonctives du roumain trouveraient des équivalents fonctionnels dans les marqueurs de structuration français tels que «eh bien », «bon», «ben», «mais» ou "donc». D’ailleurs, elle étudie aussi des "cas surmarqués » en roumain : des « expressions cumulant deux interjectifs, dont le premier 
est de règle un injonctif (interrogatif ou impératif) et le second un appellatif » (ibid., p. 31). Cette observation dégage une tendance pragmatique en roumain selon laquelle « les Roumains seraient plutôt tentés d'appeler/nommer leurs interlocuteurs d'une façon ou d'une autre, et bouclent généralement leurs syntagmes injonctifs par des vocatifs » (ibid., p. 31). Le deuxième interjectif fonctionne en roumain comme un « intensifieur » (ibid., p. 31) qui ne saurait avoir de correspondance en français: «la plupart du temps un tel marqueur est absent, ce qui prouve l'inexistence du phénomène en français » (ibid., p. 31).

L'espagnol fonctionne aussi comme le roumain de façon interjective - appellative, les FNA pouvant aussi remplir la fonction argumentative dans une conversation là où le français utiliserait des marqueurs de structuration logique.

Ce raisonnement rejoint les résultats des analyses effectuées sur mes corpus de français oral où, au milieu d'interaction, les FNA sont soit absents, soit dépourvus des rôles phatique et argumentatif. C'est pourquoi le fait de se passer des FNA en cours d'interaction adoucit significativement la force illocutoire du message et lui ôte toute trace personnelle ou émotionnelle. De cette manière, le message s'avère plus neutre et plus apte à se soumettre aux règles de la logique pure, nécessaire pour une argumentation correcte.

\section{L'apostrophe nominale de C. Détrie et le vocatif de}

\section{A. M. Bañon : deux études fonctionnelles.}

Le but de cet article est de proposer une nouvelle perspective du phénomène interpellatif français issue de la comparaisondes systèmes d'adresse espagnol et français dans un contexte interactionnel familier.

D'après les études de C. Détrie (2006) sur l'apostrophe nominale en français et celles d'A. M. Bañón (1993) sur le vocatif en espagnol, l'apostrophe ou vocatif (FNA dans les deux cas) remplirait les fonctions conative, phatique et expressive du langage définies par $\mathrm{R}$. Jakobson(1981). Transposées dans un contexte pragmatique d'interactions verbales, ces fonctions "premières » donnent lieu à des fonctions discursives variées selon leur lieu d'apparition dans l'échange communicatif. Dans l'extrait suivant, C. Détrie met à plat plutôt que la fonction première des FNA son essence même :

fonction première de l'apostrophe est donc bien d'installer dans son discours son coénonciateur, de présupposer à la fois non seulement son existence, mais aussi son identification, et donc de le positionner spatialement ( $t u$ es ici, sous mes yeux), discursivement (tu es le destinataire de mon propos), relationnellement (tu es mon ami, puisque je t'interpelle ainsi : Ami, entends-tu le vol noir des corbeaux sur nos plaines?, etc.) (2006, p. 23)

Dans l'essence des FNA il faudrait inclure à mon avis la visée conative, cette dernière étant naturellement associée à l'acte de l'appel (cf. supra). Les FNA accompagnant ou constituant à part entière des sollicitations, des pétitions, des prières, des ordres et des injonctions, risquent de jaillir à n'importe quel moment d'une interaction et, d'ailleurs, leur simple occurrence dans le discours véhicule l'accentuation de l'expressivité et l'évocation du lien interpersonnel.

C. Détrie distingue trois positions de la FNA dans l'énoncé (survenue précoce, médiane et finale) qui conditionnent sa portée pragmatique dans la situation d'énonciation. 

l'interaction verbale ou [à la] construction de la scène énonciative conditionnée par la direction de l'échange (un ou plusieurs allocutaires)» (C. Détrie, 2006, p. 84). On retiendra pour l'analyse des FMGA cette fonction de construction de la scène énonciative, et on approfondira également la fonction de la reconstruction de cette scène. En outre, on tiendra compte du fait que la sélection et surtout la discrimination d'un destinataire parmi plusieurs possibles viennent expliciter davantage la direction et le sens de l'échange, ce qui s'avère très utile notamment dans le cas des polylogues ( $\mathrm{V}$. Traverso, 1997).

Toujours en début d'interaction, G. Serbat (1987) pointe que les FNA fonctionnent aussi en tant que « acte préparatoire à la communication » et C. Détrie parle, dix ans plus tard, de leur valeur de «marqueur d'ouverture du jeu interlocutif» (2006, p. 86). De plus, au commencement d'une intervention, les FNA jouent également un rôle capital lors de l'introduction d'un énoncé au style direct.

Ce neanmoins au cours de l'interaction quand les FNA se rendent capables de faire défiler le plus grand nombre de fonctions discursives. Les FNA constituent un moyen excellent de vérification du canal communicatif (fonction phatique), puisque en appelant l'allocutaire, on sollicite son attention et on assure le maintien du contact avec lui. Ce rôle phatique peut se voir doublé d'un rôle argumentatif visant la persuasion, le rapprochement ou la distanciation de l'allocutaire, «la négociation et l'évolution des places » (Détrie, 2006, p. 118). Il s'agit de «ménager la face de l'allocutaire » (ibid.) en adoucissant ou rendant «plus acceptable un énoncé à visée perlocutoire » (ibid.). Ce rôle argumentatif (conclusif, concessif, explicatif, etc.) des FNA et l'orientation du discours qui s'en suit se font évidents en combinaison avec des interjections "eh bien», des connecteurs « mais » ou des formes, verbales « voyons, tiens », pronominales « quoi » ou adverbiales «comment» (ibid., p. 101). En fin d'interaction, les FNA fonctionnent comme " ponctuants de clôture » en indiquant la fin ou la rupture de l'interaction et en assouplissant la « régulation coénonciative » (ibid., p. 96).

41 De son côté, A. M. Bañon (1993) observe en espagnol plusieurs tendances de comportement pragmatique de la FNA suivant la position occupée par celle-ci dans l'énoncé. Ainsi «en position marginale (au début ou à la fin), la FNA porte plus sur l'énonciation que sur l'énoncé, plus sur le modus que sur le dictum étant donné sa perspective privilégiée ${ }^{3}$ » (ibid., p. 34). En position marginale-finale, A.M. Bañon constate que les FNA s'associent à d'autres marques finales, elles aussi appellatives et phatiques, et bouclent de cette façon les énoncés (cf. Liana Pop supra). Dans les salutations, les excuses, les remerciements et les énoncés injonctifs, les FNA occupent généralement la position finale.

42 En position postmarginale et prémarginale, elles peuvent remplir la fonction de connecteurs pragmatiques (cf. Détrie supra): lexèmes tels que bueno, pues, entonces et nada impliquent une évolution consécutive et conclusive par rapport au passé immédiat du discours (A.M. Bañon, 1996, p. 38).

43 Jusqu'ici, les études de C. Détrie et de A. M. Bañon montrent deux systèmes interpellatifs assez semblables par rapport à la fonction remplie par les FNA dans une position déterminée dans l'énoncé. Cependant, si plusieurs études réalisées sur l'emploi des FNA en espagnol dans un registre de langue familier attestent une forte présence de celles-ci (ou pourrait-on parler d'omniprésence?) dans n'importe quel genre d'interaction 
verbale, l'étude du même phénomène sur des corpus de français familier présente des divergences significatives -concernant le nombre et l'emploi des occurrences des FNAavec les conclusions que $\mathrm{C}$. Détrie tire de son analyse des corpus constitués de textes écrits littéraires et de discours oraux institutionnels. Les rares occurrences des FNA relevées dans l'ensemble de mes corpus ne remplissent qu'un nombre réduit des fonctions discursives étudiées par C. Détrie. Les plus récurrentes de ces fonctions semblent être la fonction conative ou de sollicitation, la fonction de marqueur d'ouverture du jeu interlocutif et la fonction d'introducteur d'un message au style direct.

Pour illustrer le comportement fonctionnel des FNA, voici trois exemples dont le premier exhibe une fonction phatique et argumentative qui fait absolument défaut dans mes corpus de français oral et familier où les interlocuteurs sont face à face.

Je vous demande, Monsieur le maire, de les consulter. (Détrie, 2006, p. 92).

Fonction phatique et argumentative.

Pika-Le-Robot> Hello lionoo, je suis content de te voir. (Corpus tchats)

Fonction de marqueur d'ouverture du jeu interlocutif.

Marine : Reyes

Angélique : Reyes

Marine: est-ce qu'on peut avoir une cuillère s'il te plait? (Corpus Marine-

Angélique)

Fonction conative, exprimant l'appel et la prière.

47 Le français familier nous fournit le reflet d'une langue parlée plus émotive, plus relâchée, qui pourrait être, comme cela se passe dans les systèmes d'interpellation d'autres langues romanes et non romanes, plus encline à l'usage des FNA. Et pourtant...il ne faut pas se fier aux a priori.

\section{Hypothèse sur la modalité mimique et gestuelle de l'interpellation comme remplaçante de l'interpellation nominale}

\subsection{Qu'est-ce qu'une forme mimo-gestuelle d'adresse (FMGA)?}

Une forme mimo-gestuelle d'adresse (FMGA) suppose la réalisation d'un appel non verbal et non vocal (cependant sa production peut être simultanée à un message verbal autre qu'une FNA) et partage avec les FNA certaines caractéristiques telles que la deixis personnelle, sociale et relationnelle et une visée conative qui lui est intrinsèque.

\subsection{Deixis personnelle et appel négligé ?}

Cosnier (1996) relève l'importance capitale de la multicanalité de la communication et de l'implication de la communication non verbale dans la transmission d'informations. Selon le type d'information transmise par la mimogestualité Cosnier classe les gestes en gestes déictiques ou désignants, en gestes illustrateurs (qui miment l'action ou figurent dans l'espace certaines caractéristiques de l'objet référent) et en gestes quasilinguistiques (qui sont des équivalents de paroles). L'observation des gestes et des mimiques déictiques ou désignants a permis de formuler « la loi de désignation du référent présent: la mention dans le discours d'un référent présent s'accompagne 
obligatoirement de sa désignation (soit par le pointage digital, soit au moins par le regard)» (ibid.).

I. Poggi et E. Magno (1998) font une nette distinction entre les gestes codifiés, " represented in the Speaker's mind as lexical items of a gestural lexicon », et les gestes créatifs, «invented on the spot to communicate some meaning via gesture ». En même temps, elles s'interrogent sur le processus générateur des gestes : « What happens when we have to indicate a referent and we do not have a codified gesture for it?» (ibid.). Finalement, la réponse qu'elles trouvent pour cette problématique est en étroite relation avec la « loi de désignation du référent présent » de Cosnier :

Creating a new gesture implies selecting one or more visually perceivable features of the referent, ones that you can imitate or just point to with your hands. Thus, one type of creative gestures is the deictic. When the referent is present in the surrounding context, its most salient feature is its present location, so that the easiest thing to do is to point at it, using hands or fingers (or nose tip, chin, gaze) to mark in which direction the Listener may find that referent. This is the origin of deictic gestures. (ibid.)

51 Si l'on transpose la loi de la désignation du référent présent et l'explication de l'origine des gestes déictiques au cas de l'interpellation mimo-gestuelle (MG), il y aura « émission d'appel » lorsque le récepteur du message est présent (physiquement ou mentalement) dans la sphère conversationnelle et coïncide avec le référent du message. Dans ce cas, il sera signalé aussi bien comme référent du message que comme destinataire de l'appel. En conséquence, l'appel émis moyennant des procédés MG sous-tendra une forte charge de deixis personnelle, relationnelle et situationnelle.

Nous concevons la deixis personnelle dans le sens premier de localisation spatiale et monstration de l'allocutaire qui est présent et partage avec le locuteur la sphère conversationnelle.

53 Par ailleurs, une FMGA constituera un appel mimo-gestuel à mi-chemin entre les gestes déictiques et les quasi linguistiques : le procédé MG mis en route montrera la personne de l'allocutaire, et sera l'équivalent non verbal des pronoms «toi/vous» ou des syntagmes nominaux en emploi interpellatif.

\subsection{Deixis relationnelle et situationnelle}

Kerbrat-Orecchioni. suggère la relative "indigence du système des marqueurs relationnels en français » (1992, p. 34), sans jamais mettre en cause son existence. Pour appuyer cet argument elle soutient qu'en français « de telles significations vont simplement se réfugier ailleurs, dans des indices plus subtils et discrets » (ibid.). Et quelques pages plus tard elle affirme que « les gestes constituent un excellent indicateur de l'état de la relation » (ibid., p. 42).

Cosnier considère lui aussi que « le toucher constitue également un signe indicateur spécial, qui peut manifester l'intimité de la relation mais aussi l'emprise et la dominance » (1996, p. 29).

Effectivement les manifestations MG laissent transparaître des attitudes que l'on peut avoir ou des sentiments ressentis pour le récepteur de notre message. On se donnera pour règle de ne pas utiliser le même signe pour interpeller un ami proche et notre chef d'entreprise. 
Pareillement, on modifiera et on adaptera la réalisation de la FMGA que l'on veut adresser à un ami proche en fonction de la situation de communication qui nous entoure. Dans un contexte déterminé on dissimulera une relation de complicité par nos manifestations MG, et dans un autre contexte, éventuellement on la simulera. On parlera de deixis situationnelle si c'est la propre manifestation MG qui nous fait prendre conscience des particularités de la sphère conversationnelle. À cet égard, Kerbrat-Orrechioni s'efforce aussi de nous rappeler qu'il faut tenir compte des «normes en vigueur dans la société considérée, et de la nature de la situation de communication » (1992, p. 36) pour réussir la réalisation et l'interprétation des signes MG. Cela s'avère essentiel dans la compréhension de certains clichés opposant la superficialité de caractère des gens du sud, généralement très tactiles, contre la profondeur des gens du nord qui ne sont pas habitués à ce rapprochement corporel quotidien et risquent de mal l'interpréter.

\subsection{FMGA et fonction conative}

La FMGA comme son homologue verbal, la FNA, est liée par la nature de l'acte de l'appel à la fonction conative. Bolinger, Heese et Trojan (1998) repèrent des « similitudes régulières de trajectoires entre les composantes vocales et gestuelles. Ainsi une montée du menton est associée à la montée de la voix dans certains questionnements». Si l'on prend en compte la forte valeur de sollicitation véhiculée par les formes d'adresse en français (cf. supra), l'assiduité avec laquelle j'ai identifié des correspondances entre la montée du menton et l'émission de l'appel dans mes corpus devient significative.

\subsection{FMGA et fonctions phatique et argumentative}

Parfois la réalisation de la FMGA cherche le consensus dans l'appel à l'autre, l'accord à propos du message énoncé. L'appel à l'allocutaire, qui se sent directement et personnellement concerné puisque inclus dans le message comme élément de l'argumentation, ne suppose qu'un rouage de plus dans le processus de la persuasion. L. Pop (cf. supra), remarque que lorsque le français utilise des marqueurs de structuration de conversation le roumain (et je me permets d'ajouter aussi le cas de l'espagnol) préfère les appels à l'écouteur pour l'inscrire dans la suite argumentative du discours et lui faire ainsi croire qu'il est pris en compte pour faire avancer le raisonnement. Souvent les FMGA sont émises en même temps qu'un terme verbal argumentatif pour appeler l'interlocuteur et attirer toute son attention au moment où s'articule la structure et s'oriente la suite logique du discours.

\section{Présentation des corpus}

\subsection{Pourquoi quatre corpus si différents?}

Cosnier soutient que "dans l'interaction conversationnelle le texte verbal subit des modifications syntaxiques selon les contraintes proxémiques imposées aux locuteurs: face à face, dos à dos, côte à côte » (1984, p. 16).

Ceci m'a donné l'idée d'observer le comportement interpellatif des français dans différents types de cadres : entre amis, avec des inconnus, face à face, sans face à face, à l'écrit, à l'oral ; cependant ces différents cadres devaient avoir au moins deux traits 
communs: les locuteurs devaient être Français, de langue maternelle française, nés et éduqués en France métropolitaine et ils devaient s'exprimer dans une langue relâchée de type familier.

Interactions sans face à face (dialogue).

\section{Interactions en face à face} hic et nunc d'une situation d'énonciation antérieure ou fictive, en réactualisant les informations sur les locuteurs, la relation interpersonnelle et le degré d'intimité existant entre eux.Dans ce sens les FMGA indirectes sont les fidèles homologues des FNA indirectes et recréent par la MG un appel fictif ou antérieur au moment de l'énonciation présente. Dans ce type de FMGA, l'interpellé est physiquement absent de la sphère conversationnelle.

\section{Analyse des corpus}

\subsection{Les chats : le comportement fonctionnel des FNA dans des polylogues écrits sans face à face}

\section{FNA et Fonction phatique}

La FNA sert à la compréhension de nombreux paramètres de la situation d'énonciation.

Dans ce sens, les chats constituent un endroit privilégié pour l'observation des informations situationnelles fournies par les FNA dont on n'a pas forcément besoin dans une situation communicative en face à face. Les FNA assurent une fonction phatique essentielle sur les chats. Dans l'exemple suivant grâce à la FNA on apprécie que Natacha 
accuse la réception du message envoyé par Sisno, ratifie que le destinataire de son message sera Sisno et explicite la nature de la relation existante entre les interlocuteurs ${ }^{4}$.

Sisno $>$ Natacha $>$ bisous tu vas

Natacha> Sisno> oui mon chouchou et toi depuis le temps

Sisno $>$ Natacha $>$ ben le chou a pousser lol

Dans les polylogues sans face à face la non-explicitation du destinataire de notre message, soit l'absence de FNA à caractère phatique, provoque des interférences dans la communication :

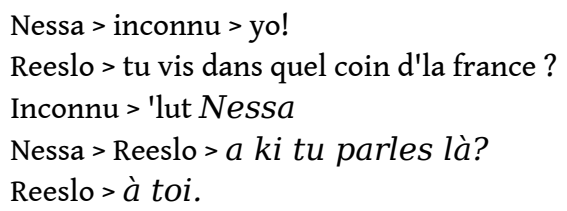

\section{FNA en survenue médiane (C. Détrie, 2006) : fonctions appellative, phatique et argumentative}

Au milieu du discours, l'introduction d'une FNA relançant un appel à notre interlocuteur implique la vérification du canal par la confirmation du destinataire et l'inclusion de celui-ci dans le déroulement logique notre discours :

rhoo-minet > il c passé Sisno que certain(es) s'amusent a dire bcp de choses sur le dos des autres, de là a parler de diffamation...

Love12 > rhoo-minet > et bien il faut chosir les personnes a qui vous parler rhoo-minet $>$ m'en fout moi Love12.. mais d'autres pas

\section{FNA autonome : fonction argumentative et explicitation de la nature du lien interpersonnel}

Par l'utilisation d'une FNA qui révèle le sentiment dominant dans la conception de l'autre, l'émetteur dirige son discours vers son destinataire et procure des charnières argumentatives basées sur la figure de l'adressé et explicite en même temps la nature du lien interpersonnel.

gaanah > coquin!

FNA liées à des formules de politesse (salutations, excuses, remerciements)

Jean-francois $>$ salut tous le monde

Love12 > Coeur $14>$ bisous

Coeur14 $>$ dsl love j ai eté coupé

venus13 > merci inconnu snif je croyais ke j'etais transparente

\section{FNA et fonction conative (prières, ordres, pétitions)}

venus13 > vous devergondez pas ced au moins?

enoAw $>$ ced raccroche

Grincheuse'> et on se calme les enfants hein...

FNA introduisant un style direct et construisant une scène énonciative fictive :

Eska $>\mathrm{y}$ a aussi le brin de muguet imaginaire

Noyel > spa cher ca

Eska > "tiens maman, j'ai pensé à t'apporter du muguet invisible" 
Selon Dimachki \& Hmed (2001), dans une conversation orale, la plupart des FNA employées dans ces exemples auraient été remplacées par un simple « tutoiement ». Cette impression et l'idée de Moscovici et Plon (1966) selon laquelle "l'énoncé verbal se rapproche de la forme écrite lorsque les sujets ne se voient pas » m'ont conduit à observer une conversation orale qui aurait deux caractéristiques, qui me semblent essentielles, en commun avec les chats: un registre de langue familier et un contexte interactionnel sans face à face. Suivant ces critères, j'ai décidé d'enregistrer une conversation de jeunes sur une station de radio de libre antenne et de lui appliquer la même analyse.

\subsection{Le mouv' (station de radio de libre antenne)}

Il s'agit d'un document audio enregistré lundi 19 mars 2007, à 23:59:34 sous format mp3. La conversation radiophonique met en scène une interaction sans tête à tête entre Emilie, animatrice de l'émission, et Ophélie, une fille qui parle de son problème avec la drogue. Dans une conversation d'environ 5 minutes, j'ai relevé seulement trois FNA liées aux fonctions discursives exposées ci-dessous :

\section{Salutation, ouverture du jeu interlocutif, inclusion d'un participant dans la scène énonciative}

euh là on sait pas si on va bien aimer quand ça s'passe comme ça parce que ça s'passe plutôt mal visiblement du côté de chez Ophélie à Dijon salut Ophélie

\section{Régulations de tours de parole}

donc on t'écoute Ophélie

\section{Fonction argumentative}

et que en plus tu n'auras plus jamais et ça tu le sais Ophélie les mêmes effets que ce que t'as eu au début.

D'après M.A. Morel, D. Bouvet et L. Danon-Boileau (1998, p. 569) :

dans la production et la réception de la parole, la modalité sonore n'est pas la seule en jeu. Recevoir la parole, ce n'est pas seulement écouter les émissions sonores de celui qui parle, c'est aussi regarder ce dernier qui déploie toute une activité corporelle tant sur le plan des gestes articulatoires nécessaires à la production phonique, que sur le plan des mouvements de la tête, des yeux, des sourcils, du buste et des mains.

Cette invitation à considérer la contribution capitale de la mimique et de la gestuelle dans le déroulement de l'interaction a été décisive pour mon travail de recherche. Ensuite, les corpus des enregistrements vidéo nous fourniront des images où l'on pourra apprécier si les fonctions pragmatiques et discursives des FNA, qui ont été évoquées plus haut, trouvent une réalisation dans la dynamique du langage corporel.

Dans le cas où les FNA feront défaut dans mes corpus, le but de mon analyse sera donc de trouver en français des synonymes discursifs (terme que j'emprunte à L. Pop, 2006) des FNA sur un plan mimique et gestuel, soit des FMGA. 


\subsection{Corpus filmés : des interactions en face à face}

\section{Les démarches de l'analyse}

Premièrement j'ai mené une analyse individuelle de chaque interlocuteur qui visait à localiser les FNA et les FMGA. Une fois localisées, j'ai procédé à l'étude de ces occurrences en contexte selon la logique suivante :

Tout d'abord j'ai réalisé la transcription du message verbal (s'il y en a un). Ensuite j'ai analysé le comportement mimique et gestuel concomitant. Pour ce faire j'ai utilisé la capture d'images du logiciel « Movie Maker».

1 Finalement j'ai essayé de dégager la fonction discursive remplie par la production de l'appel en contexte.

2 J'ai observé les appels dans le cadre de l'interaction, mais je n'ai pas entrepris l'analyse des réactions du récepteur à l'appel, et de la réussite ou l'échec de celui-ci.

Dans les corpus vidéo j’ai repéré puis analysé :

\section{4 occurrences de FNA directe}

Dans les 4 cas de FNA directe, les locuteurs ont employé les prénoms des interpellés, Sara (1), Reyes (3) n'explicitant pas la nature du lien interpersonnel. Les fonctions remplies par ces 4 FNA directe ont été notamment la fonction interpellative à visée conative. Dans le cas des FNA directe avec interpellé absent ou exclus: la volonté d'inclure celui-ci dans la sphère conversationnelle dans un but conatif constitue une autre fonction discursive à tenir en compte.

Parmi ces 4 occurrences de FNA directe, il est possible de distinguer :

1) en premier lieu, 1 occurrence de FNA directe avec interpellé dans la sphère communicativedont les traits MG les plus significatifs sont les suivants :

- Un regard fixe sur l'écouteur pendant l'émission de l'appel et une fois l'appel émis, l'émetteur baisse son regard.

- La tête de l'interpellant balaye l'espace d'énonciation, s'oriente vers l'interpellé, s'incline sur la droite pendant et après l'appel et revient sur une position centrée à la fin.

- L'interpellant étire son cou et lève son menton en direction de son écouteur. Une fois l'appel terminé, il baisse son menton.

- Son buste avance et puis, l'appel fini, son buste recule. Le bras droit se replie légèrement sur son abdomen, en guise de bouclier protégeant le corps qui s'est lancé vers l'extérieur ou qui a osé l'appel.

2) en deuxième lieu, 3 occurrences de FNA directe avec interpellé hors de la sphère communicative.

Dans le premier cas l'interpellée (moi-même) est exclue mais pas absente de la scène énonciative. L'émettrice de l'appel fixe son regard sur le mien le temps de prononcer la FNA. Avant d'appeler, elle tourne la tête à sa gauche, cherche son allocutaire et l'envisage directement. Elle incline sa tête vers l'arrière et expose son menton. Sa tête s'incline à droite pendant l'émission de l'appel.Son buste prend de l'amplitude aidé par l'écart de son bras gauche qui se déplace vers l'arrière à gauche, en ouvrant l'espace corporel vers l'interpellé en signal d'accueil, d'inclusion. L'appel fini, on assiste à l'accouplement des mains sur ses genoux qui peut être interprété comme un geste égocentré d'autoconfort. 
89 Dans le deuxième cas, où l'interpellée (moi encore) est exclue et absente de la scène énonciative, le regard de l'interpellante se pose pendant l'appel à droite sur la porte du salon, c'est-à-dire, l'endroit par où je suis sortie et où elle édifie la dernière représentation mentale qu'elle se fait de moi. Une fois l'appel émis, son regard revient à gauche sur son espace d'énonciation. Puis elle ferme les yeux. Sa tête suit avec une parfaite synchronie le mouvement de son regard tout d'abord à droite vers la porte, ensuite de retour vers le centre. Elle hausse son menton une fois l'appel verbal fini. Avant d'effectuer l'appel son buste se lance vers l'avant, son coude gauche s'écarte de son corps qui se dilate avec ce mouvement. Au moment de l'émission de l'appel son coude revient sur son corps.

\section{3 occurrences de FNA indirecte, marqueur de style direct}

90 Le support lexical choisi pour ces FNA, qui reconstruisent le hic et nunc d'une interpellation antérieure ou fictive, a été des noms explicitant une relation interpersonnelle et un certain degré d'intimité : Papa (2), doudou. Puisqu'ils doivent reconstruire une scène d'énonciation antérieure, autant donner le plus grand nombre possible d'informations la concernant. Avec ce type de FNA, on a accès à des renseignements complémentaires comme la direction et le sens de l'interpellation, l'explicitation de la relation interpersonnelle illustrant le rôle des actants (tu es mon papa, je suis ta fille; tu es mon doudou, je suis ta copine) et la nature de la situation d'énonciation antérieure (si Marine a appelé son copain doudou, elle devait être certainement dans une situation intime qui convenait à ce genre de manifestations).

91 Dans les exemples analysés les traits MG les plus curieux se déroulent juste avant de lancer l'appel : l'interpellante ferme les yeux, elle les rouvre et pose son regard au fond, en haut et à gauche (vers 22h). Elle amène mentalement son interpellé à la situation d'énonciation présente. Elle jette un coup d'œil à la représentation mentale de l'interpellé qui se trouve à gauche à côté de son allocutaire, hors la scène d'énonciation où elles se trouvent en ce moment et elle reconstruit la scène d'énonciation antérieure en s'adressant fictivement à cette représentation mentale. Ses yeux se referment. Elle profère l'appel en ayant peut-être l'image mentale de son interpellé devant elle. Sa tête juste avant et pendant l'appel s'incline énormément vers sa droite. La tête reste inclinée vers l'arrière et son menton élevé. Aucun mouvement significatif du buste n'a été repéré dans la réalisation des FNA indirecte. L'interpellante lève la paume ouverte et l'oriente vers l'extérieur de la sphère énonciative à gauche de là où elle dirige son regard. On dirait qu'elle fait un signe à quelqu'un qui se trouve derrière son écouteuse. Après, elle ramène sa main vers son corps à la hauteur de sa poitrine et ferme le poing mais laisse l'index déplié de façon déictique.

\section{FMGA directe}

Les occurrences de ce type de forme d'adresse s'élèvent dans mes corpus à près d'une centaine. Les locuteurs ne cessent pas de s'interpeller par l'intermédiaire des moyens MG. Souvent ces appels MG sont simultanés à la production verbale des mots argumentatifs (fin) ou cherchent tout simplement un consensus (c'est cool, ouai, un corps de rêve). D'autres fois, la FMGA ne s'appuie sur aucune production verbale (exemple la Kro) et dans ce cas elle pourrait être considérée comme la FMGA « pure » qui mettrait en scène un appel 100\% mimo-gestuel. 

argumentative. On observe aussi des réalisations de FMGA à moitié appellatives, à moitié argumentatives. On compare ce phénomène de convergence MG entre l'appellation et l'argumentation avec les FNA à caractère argumentatif tellement présents dans d'autres langues (roumain, espagnol) et qui manifestent un «style communicatif interjectif appellatif » (L. Pop, 2006). Le français, étant une langue moins interjective, remplacerait ce type de FNA argumentative, soit par des marqueurs de structuration au plan verbal, soit par cette forme d'adresse argumentative au plan MG.

Les traits MG les plus intéressants sont :

- avant l'émission de l'appel l'écouteur baisse son regard ou cherche l'espace partagé de l'énonciation. Pendant l'interpellation les yeux sont fixés sur l'interpellé et une fois l'appel émis, ils se referment.

- la tête cherche son interlocuteur (à droite ou à gauche), se lance en avant, commence à se hausser et s'incline en arrière en exposant le menton. Après, la tête redescend et récupère sa position initiale centrée en hauteur.

- le buste s'ouvre et s'offre à l'allocutaire, se penche vers l'avant pendant l'émission de l'appel et, après celle-ci, il s'éloigne dans la direction contraire à celle de l'interlocuteur. Dans un exemple, l'interpellante baisse le bras qu'elle avait utilisé pour effectuer l'appel et qu'elle utilise maintenant pour se protéger du regard de l'autre, pouvant ainsi redresser son buste.

- tous ses doigts se replient sur la paume de sa main, sauf l'index (doigt déictique par excellence) qu'elle tient droit et qu'elle utilise pour signaler et identifier le récepteur de son message et pour effectuer le signal d'appel.

- elle tape son co-énonciateur du doigt plusieurs fois. Ensuite elle le retire pour venir le poser sous son nez dans un geste égocentré.

\section{FMGA indirecte}

J'en ai localisé une seule occurrence. Très illustrative certes, basée sur un mot argumentatif (ben) et un autre mot axiologique (parfait). La reconstruction hic et nunc d'une FMGA émise dans une scène énonciative antérieure semble évidente dans cet exemple. Les caractéristiques MG les plus symboliques de la FMGA indirecte sont le déplacement du regard en haut et à gauche juste à côté de l'allocutaire, en position de 10h. Comme cela se passe pour les FNA indirecte, l'interpellante reconstruit la scène d'énonciation antérieure et pose son regard sur la représentation mentale de son interlocuteur imaginaire dans la situation d'énonciation actuelle. Une fois l'interpellation recréée, son regard revient sur l'allocutaire présente.

Elle incline sa tête vers la droite et vers l'arrière et montre son menton. L'appel émis, sa tête revient sur l'allocutaire. Son buste se hausse légèrement pour accompagner son geste. Elle lève sa main droite qui reste ouverte, les doigts étirés. Puis, seul son index reste complètement étiré et les autres doigts sont repliés et s'orientent vers la paume. C'est le geste de deixis personnelle qu'on trouve dans d'autres exemples de FMGA. Elle signale son interlocuteur imaginaire. Elle abandonne la forme déictique adoptée par sa main et descend sa main jusqu'à ce que celle-ci se pose à nouveau sur sa tasse. 


\section{Conclusions}

\subsection{Nombre et nature fonctionnelle des FNA et FMGA}

\section{Interactions sans face à face}

\section{Sur les chats}

On assiste à la prolifération et à l'épanouissement fonctionnel des FNA sur les chats. Les occurrences et les emplois des FNA se multiplient dans ce genre d'interaction plurilocuteurs sans face à face. Elles intègrent une technique d'accueil visant l'inclusion d'un participant externe dans la sphère d'énonciation. Elles mettent en place également d'autres stratégies discursives (phatique, argumentative, expressive et d'introduction du style direct) qui assouplissent l'échange verbal dans une situation de communication démunie, à proprement parler, d'informations contextuelles essentielles. Pour combler au maximum ce manque, tout type de lexème a été employé : des simples pseudos aux termes d'affection, de haine, de relation de parenté, etc.

\section{À la radio}

On atteste l'emploi des FNA liées aux formules de salutation et d'inclusion d'un intervenant extérieur, à la régulation du tour de parole et à des buts phatique / argumentatif. Le fait de ne trouver que 3 FNA dans une interaction orale de 5 minutes sans face à face me semble être, par comparaison avec l'espagnol, d'une réelle indigence appellative. D'ailleurs, la FNA reprend dans les trois cas le prénom Ophélie (son allocutaire), c'est-à-dire, une forme neutre qui n'explicite d'aucune manière la nature de la relation interpersonnelle. Les seules indications que le prénom apporte sur l'interpellé sont, d'un côté sa ratification comme destinataire de notre appel, d'un autre côté son identification et discrimination parmi d'autres (mon destinataire s'appelle X).

\section{Interactions en face à face}

Sur environ une heure de conversation (dialogue et polylogue confondus) on a constaté une réduction significative des occurrences et des emplois des FNA par rapport aux interactions sans face à face. Cette diminution pourrait être associée au fait de partager la même situation d'énonciation et donc d'avoir recours aux mêmes informations contextuelles nécessaires au cadrage et au bon déroulement de l'interaction. Parmi ces informations nécessaires on peut compter les informations véhiculées par la communication mimo-gestuelle. Dans le face à face, un simple « qui parle à qui » peut être facilement clarifié par un mouvement de la tête, du menton ou même du buste en direction de l'interpellé. Par conséquent on observe une nette augmentation du nombre des FMGA au détriment des occurrences de FNA.

D'un autre côté, les FMGA présentent des traits MG récurrents et communs avec les FNA :

- La grande charge déictique de la FMGA «deixis personnelle, relationnelle, situationnelle » implique souvent la réalisation du geste déictique par excellence : la monstration de l'interpellé avec l'index étiré et les autres doigts repliés sur la paume de la main. 
- La main qui s'ouvre et qui s'offre à l'interpellé avec les bouts des doigts dans sa direction (le degré d'inclinaison de la paume vers le haut ou vers le centre, pouvant être un indice d'argumentation) constitue un autre geste assidûment analysé.

- La montée du menton et son orientation vers l'interpellé, ainsi que l'ouverture, l'orientation et le rapprochement du buste ont été aussi classés comme des manifestations MG récurrentes.

- D'ailleurs, comme M.-A. Morel et D. Bouvet (2002) l'avaient déjà noté, l'appel s'accompagne habituellement d'un « regard fixe et direct vers le destinataire et d'une nette avancée de la tête et du buste dans sa direction ». Ce regard s'oriente vers l'extérieur de la scène conversationnelle à $22 \mathrm{~h}$ quand il s'agit de reconstruire un appel antérieur ou fictif.

- Ce qui est aussi assez curieux c'est la protection que l'interpellant assure de son corps face à l'acte de «menace » (dans le sens d'invasion expliqué plus haut) de l'appel. L'appel lancé, l'interpellant retourne sur un espace de sécurité égocentré notamment grâce à des bras qui se placent devant le corps en sorte de bouclier, des mains qui s'accouplent sur les genoux, des mouvements de recul du buste, de la tête, le fait de fermer les yeux ou de baisser le regard montrent à quel point on craint la réaction de l'autre face à l'acte de l'appel.

\section{BIBLIOGRAPHIE}

BAÑON, A.M. (1993). El Vocativo en Español : Propuestas para su análisis lingüístico. Barcelona : Octaedro.

BOUVET, D., \& MOREL, M.-A. (2002). Le ballet et la musique de la parole. Paris : Ophrys.

CALBRIS, G. (2003). L'expression gestuelle de la pensée d'un homme politique. Paris: CNRS.

COSNIER, J., \& BROSSARD, A. (1984). La communication non verbale. Neuchâtel : Delachaux et Niestlé.

COSNIER, J. (1996). Les gestes du dialogue, la communication non verbale. Psychologie de la motivation, 21, 129-138.

BOUVET, D., MOREL, M.-A., \& DANON-BOILEAU, L. (1998). Se parler, c'est aussi se regarder et se donner à voir. In Santi S., Guaïtella I., Cavé C., Konopczynski G. (éds), ORAGE'98, ORAlité et Gestualité: communication multimodale, interaction, Paris : L'Harmattan, 569-575.

DETRIE, C. (2006). De la non-personne à la personne : L'apostrophe nominale. Paris : CNRS.

GOFFMAN, E. (1974). Les rites d'interaction. Paris : Minuit.

JAKOBSON, R. (1981). Essais de linguistique générale. Paris : Minuit.

KERBRAT-ORECCHIONI, C. (1992). Les interactions verbales (t. 2). Paris : Armand Colin.

KERBRAT-ORECCHIONI, C. (éd.). (2010 à paraître). S'adresser à autrui. (Vol. 1) : Les Formes Nominales d'Adresse dans les interactions orales en français. Presses universitaires de Chambéry.

KERBRAT-ORECCHIONI, C. (éd.). (2011 en préparation). S'adresser à autrui. (Vol. 2) : Les Formes Nominales d'Adresse dans une perspective interculturelle. Presses universitaires de Chambéry. 
LEON MIRANDA, R. (2008). C'est le geste qui compte. Mémoire de Master 2 non publié, Université Paris 3, Paris.

LEON MIRANDA, R. (2011 en préparation). Les FNA dans les conversations entre jeunes : Comparaison français/espagnol. In Kerbrat-Orecchioni (éd.) Presses universitaires de Chambéry.

MOSCOVICI, S., \& PLON, M. (1966). Les situations colloques. Observations théoriques et expérimentales. Bulletin de Psychologie, 19, 702-722.

POGGI I., \& MAGNO E. (1998). A procedure for the generation of gestures in bimodal communication. In Santi S., Guaïtella I., Cavé C., Konopczynski G. (éds), ORAGE'98, ORAlité et Gestualité: communication multimodale, interaction. Paris : L'Harmattan, 201-209.

POP, L. (2006). L'interjection : Jeux et enjeux. Langages, 161, 24-36. Paris : Armand Colin.

SELESKOVITCH, D., \& LEDERER, M. (2001). Interpréter pour traduire. Paris : Didier Erudition.

SERBAT, G. (1987). Sur le vocatif. Le vocatif : un acte de parole. Vita Latina, 106, 7-13. Avignon : Aubanel.

TRAVERSO, V. (1997). Des échanges à la poste : dilogues, trilogues, polylogue(s). Cahiers de Praxématique 28, 57-77.

WAGNER, R.-L., \& PINCHON, J. (1991). Grammaire du français classique et moderne. Paris : Hachette.

DIMACHKI, L. \& HMED, N. (2001). “Bonjour madame !", “Bonjour mon frère !”: Le système des termes d'adresse dans des interactions verbales en France, au Liban et en Tunisie. Retrieved August 19, 2009, fromhttp://icar.univ-lyon2.fr/documents/gric1/Bonjour_madame.rtf

ENAJAS, R. (2004, June). El vocativo amoroso en el lenguaje juvenil almeriense.Tonos: Revista electrónica de estudios filológicos, № 7. Retrieved August 28, 2009, fromhttp:// dialnet.unirioja.es/servlet/articulo?codigo $=919463$

\section{NOTES}

1. Équipe constituée dans le cadre du laboratoire ICAR (CNRS-Université Lyon 2) et soutenue par l'ILF.

2. Traduction par moi-même.

3. J'ai traduit la citation espagnole en français.

4. Les FNA ont été transcrites en italique et la direction de l'échange se manifeste à l'écrit selon ce schéma: Pseudo du locuteur + symbole de direction et sens du message >+ Pseudo du destinataire.

\section{RÉSUMÉS}

À partir d'un corpus constitué de trois enregistrements oraux et d'un corpus écrit, cette étude vise un double objectif : premièrement, il s'agira d'analyser les occurrences des formes nominales d'adresse (Kerbrat-Orecchioni, 2010, à paraître) du point de vue de leur(s) fonction(s) pragmatique(s) dans le discours et de leur réalisation mimique et gestuelle. Ensuite, il sera 
question de présenter et d'analyser les formes mimo-gestuelles d'adresse qui, n'ayant pas de support nominal dans le discours, constituent d'elles-mêmes un appel au même titre que les FNA et remplissent les mêmes fonctions pragmatiques, voire plus, que celles-ci.

My study is based on three oral corpora and one written corpus and it has a double objective. Firstly, nominal forms of address (FNA) (Kerbrat-Orecchioni, 2010, in press) will be analysed from the point of view of their pragmatic function(s) within the speech and their facial and gestural realisation. Secondly, facial and gestural forms of address (FMGA) will be introduced and analysed. These FMGAdo not have a nominal support in speech but represent a real way of addressing that fills the same, or even more, pragmatic functions as the FNA.

\section{INDEX}

Mots-clés : formes nominales et mimo-gestuelles d'adresse, fonctions pragmatiques

Keywords : facial and gestural forms of address, pragmatic functions

\section{AUTEUR}

\section{REYES LEON MIRANDA}

Université Paris 3 